Homology, Homotopy and Applications, vol.15(1), 2013, pp.303-311

\title{
CONNECTIVITY AT INFINITY FOR BRAID GROUPS ON COMPLETE GRAPHS
}

\author{
JOHN MEIER AND LIANG ZHANG
}

(communicated by Nathalie Wahl)

\begin{abstract}
We show that the connectivity at infinity for configuration spaces on complete graphs is determined by the connectivity of chessboard complexes.
\end{abstract}

\section{Introduction}

The recent interest in braid groups on graphs was sparked by work of Abrams and Ghrist $[\mathbf{1}, \mathbf{2}, \mathbf{1 3}]$. In addition to highlighting the connection to robot motion planning, these papers present non-positively curved classifying spaces for these groups. One of the striking, elementary examples of this construction is that the space for two robots on the complete graph $K_{5}$ is the closed orientable surface of genus 6. Early work on these classifying spaces established basic topological properties, such as their Euler numbers $[\mathbf{1 1}]$ and presentations of their fundamental groups $[\mathbf{9}, \mathbf{1 7}]$. Recent progress has, however, found it necessary to focus on special cases in order to get concrete topological results. As a case in point, the computation of integral homology and cohomology rings was undertaken in the case where the underlying graphs are trees $[\mathbf{8}, \mathbf{1 0}]$ and for 2-braid groups on planar graphs [4].

In this paper we work with something of the opposite extreme from trees and planar graphs and determine the connectivity at infinity of braid groups on complete graphs. Connectivity at infinity is essentially the study of connectivity properties that persistently occur in the complements of compact sets. For example, $\mathbb{R}^{n}$, for $n \geqslant 2$, is 0 -connected at infinity (that is, one-ended) and in general $\mathbb{R}^{n}$ is $(n-2)$-connected at infinity. Connectivity at infinity is a topological invariant of a group, and it has important implications from group cohomology to manifolds. If a group $G$ admits a finite, $m$-dimensional classifying space $K=K(G, 1)$, then the universal cover $\widetilde{K}$ is at best $(m-2)$-connected at infinity; if $\widetilde{K}$ is $(m-2)$-connected at infinity, this implies that $G$ is an $m$-dimensional duality group (see [12]).

As a corollary to our main theorem, we show that:

Corollary 1.1. Let $n \geqslant m \geqslant 2$. The group of $m$-robots on a complete graph $K_{m+n}$ is an m-dimensional duality group if and only if $n \geqslant 2 m-1$.

Our paper is structured in the following way: Section 2 provides background information about configuration spaces for graphs, connectivity at infinity, and local-to-

Received October 24, 2012, revised March 22, 2013; published on May 31, 2013.

2000 Mathematics Subject Classification: 20F65, 57M07.

Key words and phrases: braid group on a graph, connectivity at infinity.

Article available at http://intlpress.com/HHA/v15/n1/a15 and doi:10.4310/HHA.2013.v15.n1.a15

Copyright (C) 2013, International Press. Permission to copy for private use granted. 
asymptotic theorems for CAT(0) cubical complexes. Section 3 establishes the connection between the link of a vertex in configuration space on a complete graph and the well-known chessboard complexes. We prove the main result about connectivity at infinity for braid groups on complete graphs in Section 4. The key step in our argument uses established properties of chessboard complexes and the relationship between connectivity at infinity and connectivity of links of vertices for CAT(0) cubical complexes.

\section{Background}

\subsection{The classifying space for the group of robots on a graph}

Definition 2.1. Let $G$ be a finite, connected, simple graph, viewed as a simplicial complex. Let $\sigma$ be vertex or edge of $G$. Define $\partial \sigma$ to be $\sigma$ when $\sigma$ is a vertex, and $\partial \sigma$ to be its two vertices when $\sigma$ is an edge. Fix a natural number $m$. The discretized $m$ point configuration space on $G, C_{m}(G)$, is the subcomplex of $\underbrace{G \times \cdots \times G}_{m \text { copies }}$ consisting of the product cells $\sigma_{1} \times \cdots \times \sigma_{m}$ satisfying $\partial \sigma_{i} \cap \partial \sigma_{j}=\emptyset$ for all $i \neq j$.

One might view $C_{m}(G)$ as follows: Suppose $m$ labelled robots are positioned at distinct vertices of $G$. Each robot may move from its vertex to an adjacent vertex by traveling along an edge, with the caveat that a robot cannot travel along an edge if there is another robot at one of the edge's vertices. Under this restriction, the set of allowed configurations of the robots is $C_{m}(G)$. The fundamental group of $C_{m}(G)$ describes the collection of all allowed motions of these robots, beginning and ending in a fixed position. Because of the analogy with the pure braid groups, these groups are often referred to as pure braid groups on graphs.

The fundamental group of $C_{m}(G)$ is often called the group of $m$ robots on $G$ or the $m$-strand pure braid group on $G$.

The space $C_{m}(G)$ comes with a natural cubical structure. Vertices of $C_{m}(G)$ correspond to configurations where all the robots are located at vertices of $G$; edges of $C_{m}(G)$ correspond to a single robot moving along an edge of $G$; squares in $C_{m}(G)$ correspond to two robots moving along two edges in $G$, and so on. (See Figure 1.) The fact that the resulting cubical complex is locally $\mathrm{CAT}(0)$, that is, it satisfies the well-known link condition, was established by Abrams [1]:

Theorem 2.2. The space $C_{m}(G)$ is a locally $\mathrm{CAT}(0)$ cube-complex. Hence $C_{m}(G)$ is a $K(\pi, 1)$.

Remark 2.3. Because our arguments are combinatorial, we are working with the combinatorial or discretized definition for configuration spaces on a graph. One could take a topological perspective, working with the space:

$$
C_{m}^{\mathrm{top}}(G)=\left\{\left(x_{1}, \ldots, x_{m}\right) \mid x_{i} \neq x_{j} \text { if } i \neq j\right\} \subset G \times \cdots \times G .
$$

Abrams proved that given any $m$ and $G$ there is a subdivision of $G, G^{\prime}$, such that $C_{m}^{\text {top }}(G)$ deformation retracts onto $C_{m}\left(G^{\prime}\right)$. 

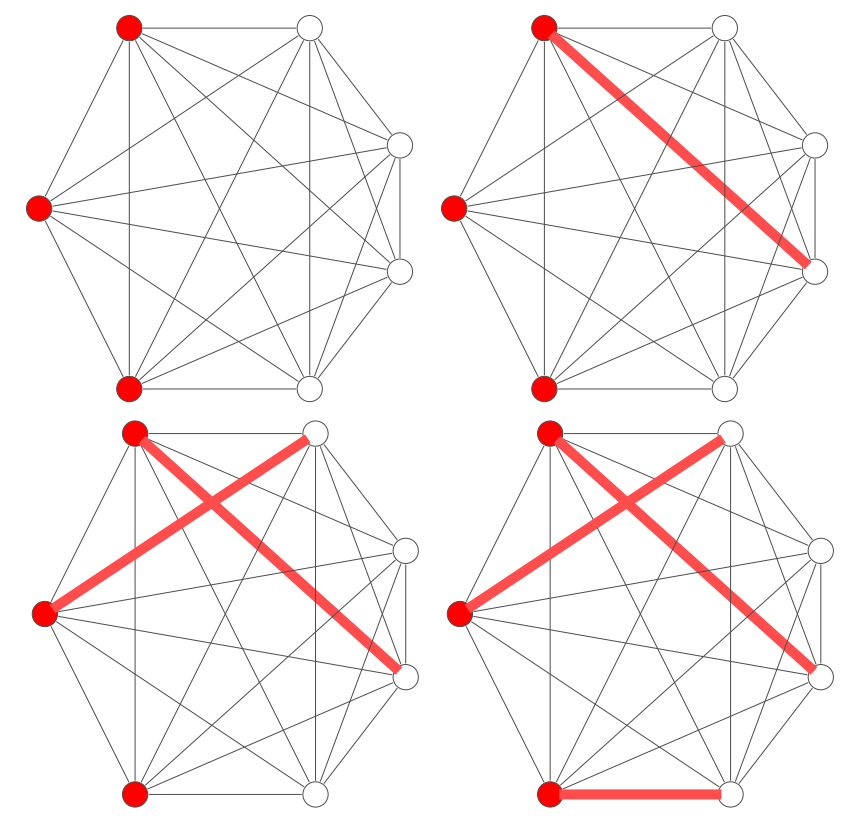

Figure 1: An example of a vertex, edge, square, and cube in $C_{3}\left(K_{7}\right)$. A thickened edge denotes the path travelled by a robot.

\subsection{Topology at infinity for CAT $(0)$ cube complexes}

Because of the connection to the classical braid groups and because some wellknown examples of the complexes $C_{m}(G)$ are surfaces or pseudo-manifolds, it is natural to wonder if there are general situations where the complexes $C_{m}(G)$ exhibit manifold-like behavior.

Definition 2.4. Let $\widetilde{X}$ be the universal cover of a finite, aspherical, CW complex $X$. We say $\widetilde{X}$ is $m$-connected at infinity if given any compact $C \subset \widetilde{X}$ there is a compact $D \supset C$ such that any map $\phi: S^{n} \rightarrow \widetilde{X}-D$ extends to a map $\widehat{\phi}: B^{n+1} \rightarrow \widetilde{X}-C$ for all $-1 \leqslant n \leqslant m$.

Since being (-1)-connected is the same as being non-empty, the property of being $(-1)$-connected at infinity is equivalent to saying $\tilde{X}$ is infinite. The property 0 -connected at infinity is often called one-ended, and 1-connected at infinity is often called simply-connected at infinity. If the complex $\widetilde{X}$ is a manifold of dimension $d \geqslant 4$, then work of Stallings and Freedman shows that being simply connected at infinity is both necessary and sufficient for establishing that $\widetilde{X}$ is homeomorphic to $\mathbb{R}^{d}$.

Connectivity at infinity is an invariant of the fundamental group. That is, let $X$ and $Y$ be two $K(G, 1)$ s (with $G$ a fixed group). Then if the universal cover $\widetilde{X}$ is $m$-connected at infinity, then so is the universal cover $\tilde{Y}$. There is also a notion of acyclic at infinity, where one replaces the homotopical condition in the definition above with the corresponding homological condition. This homological version is then 
equivalent to the vanishing of cohomology with compact supports, which is the standard way to define the notion of a duality group. An excellent, detailed discussion of connectivity at infinity and related notions can be found in [12].

We use the following result from $[\mathbf{7}]$ :

Theorem 2.5. Let $X$ be a finite, locally CAT(0) cube-complex with universal cover $\widetilde{X}$. If the link of every vertex in $X$ is $n$-connected, and remains $n$-connected when any closed simplex is removed, then $\widetilde{X}$ is n-connected at infinity.

There is a partial converse to this statement, originally presented in the context of general CAT(0), piecewise-Euclidean complexes in $[6]$. In the context of a cube complex, Corollary 5.3 of that paper implies the following result:

Theorem 2.6. Let $X$ be a finite, locally CAT(0) cube-complex with universal cover $\widetilde{X}$, and fix an integer $n, n \geqslant-1$. Assume the link of every vertex in $X$ is $n$-connected, and remains $n$-connected when any closed simplex is removed. If the link of some vertex $v$ has $H_{n+1}(\operatorname{LK}(v)) \neq 0$, then $\widetilde{X}$ is not $(n+1)$-connected at infinity.

When $n=-1$ this result provides a local method of establishing that $\widetilde{X}$ is not one-ended. First it must be the case that the link of every vertex is non-empty and remains non-empty when any closed simplex is removed. Then, if there is a vertex with a disconnected link (a link that is not 0 -connected), it follows that $\widetilde{X}$ is not 0 -connected at infinity.

\section{Links in $C_{m}\left(K_{m+n}\right)$ and chessboard complexes}

In the remainder of this paper we study $C_{m}\left(K_{m+n}\right)$, where $K_{m+n}$ denotes the complete graph on $m+n$ vertices. The central question is to understand the connectivity at infinity of the universal cover of $C_{m}\left(K_{m+n}\right)$, denoted by $C_{m} \widetilde{\left(K_{m+n}\right)}$. Theorems 2.5 and 2.6 connect the connectivity at infinity of $C_{m} \widetilde{\left(K_{m+n}\right)}$ to connectivity properties of links of vertices. For this reason we focus on the link of a vertex in $C_{m}\left(K_{m+n}\right)$, which we denote by $\operatorname{LK}(m, n)$. By symmetry, the links of all vertices in $C_{m}\left(K_{m+n}\right)$ are isomorphic, so the notation is not ambiguous.

A permissible placement of rooks on an $m \times n$ chessboard is any placement in which no two rooks are in the same row or in the same column. The set of all permissible placements forms a partially ordered set, with the order being given by containment. The simplicial realization of this poset is denoted by $\Delta_{m, n}$. The same complex can be constructed as the matching complex on the complete bipartite graph $K_{m, n}$. The elements of the underlying poset are edges of $K_{m, n}$ that "match" vertices in the two parts of the bipartite graph, and the ordering is again by containment. The isomorphism between these two complexes is illustrated in the middle and rightmost picture in Figure 2.

Lemma 3.1. The link of any vertex in $C_{m}\left(K_{m+n}\right)$ is isomorphic to $\Delta_{m, n}$.

Proof. In $C_{m}\left(K_{m+n}\right)$, let $v$ be the vertex associated with the configuration, where the robots are positioned at the vertices $r_{1}, \ldots, r_{m}$ and the available vertices are $f_{1}, \ldots, f_{n}$. 
A vertex in the link of $v$ corresponds to a movement where a robot moves from $r_{i}$ to $f_{j}$ while the remaining $m-1$ robots stay still. We denote this by $r_{i} \mapsto f_{j}$. So the vertex set of $\operatorname{LK}(v)=\left\{r_{i} \mapsto f_{j} \mid 1 \leqslant i \leqslant m, 1 \leqslant j \leqslant n\right\}$. A set of vertices $\left\{r_{i_{1}} \mapsto\right.$ $\left.f_{j_{1}}, \ldots, r_{i_{t}} \mapsto f_{j_{t}}\right\}$ forms a simplex in $\operatorname{LK}(v)$ if $i_{p} \neq i_{q}$ and $j_{p} \neq j_{q}$ when $p \neq q$. The function that replaces $r_{i} \mapsto f_{j}$ by $(i, j)$ provides an isomorphism with the chessboard complex $\Delta_{m, n}$.
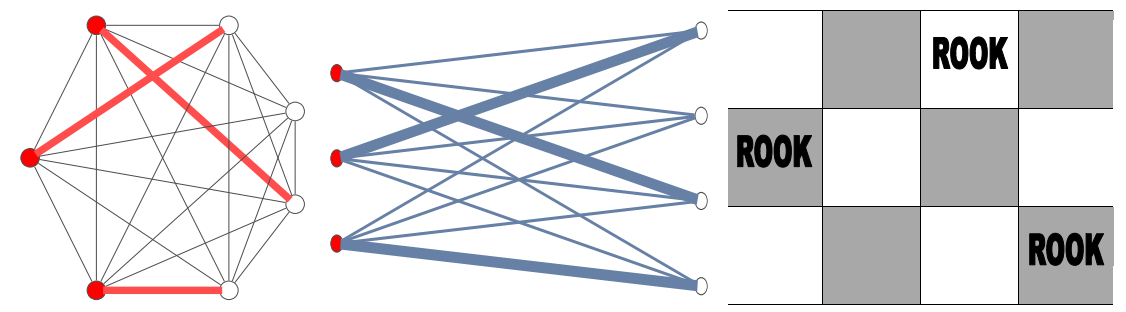

Figure 2: A 2-simplex in $\operatorname{LK}(3,4)$ and its associated 2-simplices in the chessboard and matching complexes.

Example 3.2. Figure 2 indicates three representations of the same 2 -simplex in the link of a vertex in $C_{3}\left(K_{7}\right)$ : as coming from a cube in $C_{3}\left(K_{7}\right)$, as a 2-simplex in the matching complex of $K_{3,4}$ and as a 2 -simplex in $\Delta_{3,4}$. We note that $\Delta_{3,4}$ is a torus, and therefore that $C_{3}\left(K_{7}\right)$ is a pseudo-3-manifold where the links of vertices are tori.

The connectivity degree of chessboard complexes has been an interesting question for combinatorialists. Björner, Lovász, Vrećica and Živaljević proved a connectivity bound [5], which was eventually shown to be sharp (see [18] and [19]). The result is:

Theorem 3.3. Let $v_{m, n}=\operatorname{Min}\left\{m, n,\left\lfloor\frac{m+n+1}{3}\right\rfloor\right\}$. Then $\Delta_{m, n}$ is $\left(v_{m, n}-2\right)$-connected but not $\left(v_{m, n}-1\right)$-connected.

Remark 3.4. It should be noted that the topology of the chessboard complex $\Delta_{m, n}$ is not as simple and intuitive as its rather elementary definition might suggest. It is known that torsion can be present in the integral homology of these spaces. For example, $H_{2}\left(\Delta_{5,5} ; \mathbb{Z}\right)=\mathbb{Z}_{3}$. (See $[\mathbf{1 4}, \mathbf{1 5}, \mathbf{1 6}, \mathbf{1 8}, \mathbf{1 9}]$ and the references cited there.)

\section{Connectivity at infinity for braid groups on complete graphs}

We cannot yet bundle together the local-to-asymptotic result quoted in Theorem 2.5 with the connectivity result quoted in Theorem 3.3 to immediately establish our main result, Theorem 4.8. Theorem 2.5 requires not only that the links of vertices have certain connectivity properties, but also the punctured links need to be sufficiently connected in order to ensure connectivity at infinity. In the remainder of this section we explain how results in the combinatorics literature establish the necessary conditions. 
Let $\Delta_{m . n}-\sigma$ denote the simplicial complex obtained when a closed simplex $\sigma$ is removed from $\Delta_{m, n}$. Here we allow the possibility that the simplex removed could be the empty simplex. We note that there is an action by the product of symmetric groups $\Sigma_{m} \oplus \Sigma_{n}$ on $\Delta_{m, n}$ that is transitive on $k$-simplices for any fixed $k$. This action induces a combinatorial bijection between $\Delta_{m, n}-\sigma$ and $\Delta_{m, n}-\sigma^{\prime}$ whenever $\sigma$ and $\sigma^{\prime}$ have the same dimension.

Our approach to establishing the connectivity properties of punctured links is to examine one approach to establishing connectivity properties of the chessboard complexes. This approach focuses on the notion of vertex decompositions of simplicial complexes.

Definition 4.1. A simplicial complex $S$ is vertex decomposable if it is pure and it is either empty, or it has a vertex $v$ such that $S-v$ and $\operatorname{LK}(v)$ are both vertex decomposable.

Note that if $S$ is vertex decomposable, then there is a sequence of vertices $\left\{v_{1}, v_{2}, \ldots\right\}$ such that $S, S \backslash\left\{v_{1}\right\}, S \backslash\left\{v_{1}, v_{2}\right\}$, etc. are all vertex decomposable. This property is of interest because of the following well-known chain of implications:

Vertex Decomposable $\Rightarrow$ Shellable $\Rightarrow$ Homotopy Cohen-Macaulay.

A simplicial complex $S$ is homotopy Cohen-Macaulay if given any simplex $\sigma \subset S$, including $\sigma=\emptyset, \operatorname{LK}(\sigma)$ is $(\operatorname{Dim}(\operatorname{LK}(\sigma))-1)$-connected.

We will make frequent use of the following lemma, which is immediate from the definition of vertex decomposability:

Lemma 4.2. Let $S$ be a simplicial complex that is vertex decomposable. If the decomposition begins with $v_{1}, v_{2}, v_{3}, \ldots$ and $\left\{v_{1}, \ldots, v_{k}\right\}$ span a simplex $\sigma \subset S$, then $S \backslash \sigma$ is vertex decomposable. Further, $S \backslash \sigma$ is homotopy Cohen-Macaulay.

In order to extend the connectivity results from $\Delta_{m, n}$ to $\Delta_{m, n}-\sigma$, we will use the following results-Theorems 4.3 and 4.5 - about chessboard complexes (see [3] and $[\mathbf{2 0}])$ :

Theorem 4.3. Let $m \geqslant 2$ and $n \geqslant 2 m-1$. Then $\Delta_{m, n}$ is vertex decomposable.

The proof of this result in [20] constructs a fairly explicit vertex decomposition of $\Delta_{m, n}$. For $1 \leqslant i \leqslant m$, the $i$-th vertex in the ordering is $(m+1-i, 2(m+1-i)-1)$. Thus if $\sigma$ is a $k$-dimensional simplex, we may by symmetry assume

$$
\sigma=\{(m, 2 m-1),(m-1,2 m-3), \ldots,(m-k, 2 m-2 k-1)\} .
$$

It follows from the definition of vertex decomposability that $\Delta_{m, n}-\sigma$ is vertex decomposable. Using Lemma 4.2 we get

Corollary 4.4. Let $m \geqslant 2, n \geqslant 2 m-1$ and suppose $\sigma$ is any simplex in $\Delta_{m, n}$, possibly the empty simplex. Then $\Delta_{m, n}-\sigma$ is vertex decomposable.

Theorem 4.5. Let $2 m-2 \geqslant n \geqslant m \geqslant 2$ and $k=\left\lfloor\frac{m+n+1}{3}\right\rfloor$. Then $\Delta_{m, n}^{(k-1)}$ is vertex decomposable. 
Similar to the argument above, we may use the vertex decomposition of $\Delta_{m, n}^{(k-1)}$, as explained in [3], to show that for any simplex $\sigma \subset \Delta_{m, n}^{(k-1)}, \Delta_{m, n}^{(k-1)}-\sigma$ is vertex decomposable. The essential fact is that the initial vertices used in the vertex decomposition are the vertices describing a maximal dimensional simplex in the complex. Again by Lemma 4.2 we get

Corollary 4.6. Let $2 m-2 \geqslant n \geqslant m \geqslant 2$ and $k=\left\lfloor\frac{m+n+1}{3}\right\rfloor$. Suppose $\sigma$ is any simplex in $\Delta_{m, n}$, possibly the empty simplex. Then $\Delta_{m, n}^{(k-1)}-\sigma$ is vertex decomposable.

Theorem 4.7. Let $m, n \geqslant 2$ and $v_{m, n}=\operatorname{Min}\left\{m, n,\left\lfloor\frac{m+n+1}{3}\right\rfloor\right\}$. Let $\sigma$ be any simplex in $\Delta_{m, n}$. Then $\Delta_{m, n}-\sigma$ is $\left(v_{m, n}-2\right)$-connected.

Proof. By symmetry, assume $n \geqslant m$.

If $n \geqslant 2 m-1$, then $v_{m, n}=m$. By Corollary $4.4, \Delta_{m, n}-\sigma$ is vertex decomposable and thus Cohen-Macaulay, which implies that $\widetilde{H}_{i}\left(\Delta_{m, n}-\sigma\right)$ vanishes for $i \leqslant m-2$. Therefore, $\Delta_{m, n}-\sigma$ is $(m-2)$-connected.

If $n \leqslant 2 m-2$, let $k=\left\lfloor\frac{m+n+1}{3}\right\rfloor=v_{m, n}$. By Corollary 4.6, $\Delta_{m, n}^{(k-1)}-\sigma$ is vertex decomposable and thus Cohen-Macaulay. Consequently, for $i \leqslant k-2, \widetilde{H}_{i}\left(\Delta_{m, n}^{(k-1)}-\right.$ $\sigma)=0$. As a result, $\Delta_{m, n}^{(k-1)}-\sigma$ is $(k-2)$-connected, so $\Delta_{m, n}-\sigma$ is $(k-2)$-connected.

We now state our main result.

Theorem 4.8. Let $m, n \geqslant 2$, let $d=\operatorname{Min}\{m, n\}$, and let

$$
v_{m, n}=\operatorname{MiN}\left\{m, n,\left\lfloor\frac{m+n+1}{3}\right\rfloor\right\} \text {. }
$$

Then $C_{m} \widetilde{\left(K_{m+n}\right)}$ is a d-dimensional complex that is $\left(v_{m, n}-2\right)$-connected at infinity but not $\left(v_{m, n}-1\right)$-connected at infinity.

Proof. The claim about the dimension of $\widetilde{C_{m}} \widetilde{\left(K_{m+n}\right)}$ is immediate from the construction of $C_{m}\left(K_{m+n}\right)$. The claim that $C_{m}\left(K_{m+n}\right)$ is $\left(v_{m, n}-2\right)$-connected at infinity follows from Theorem 2.5, together with Lemma 3.1, Theorem 3.3, and Theorem 4.7. The claim that $C_{m} \widetilde{\left(K_{m+n}\right)}$ is not $\left(v_{m, n}-1\right)$-connected at infinity follows from Theorem 2.6, together with Lemma 3.1, Theorem 3.3, and Theorem 4.7.

We can now restate the result highlighted in the introduction.

Corollary 4.9. Let $n \geqslant m \geqslant 2$. Then the group of $m$ robots on $K_{m+n}$ is an $m$-dimensional duality group if and only if $n \geqslant 2 m-1$.

Remark 4.10. Note that there is a complete symmetry between the vertices occupied by the robots and the unoccupied vertices, implying a symmetry between the roles of $m$ and $n$. This explains, in particular, the symmetry in Theorem 4.8. Similarly, it can be used to derive the next result, which is something of a dual of Corollary 4.9.

Corollary 4.11. Let $m \geqslant n \geqslant 2$. Then the group of $m$ robots on $K_{m+n}$ is an $n$-dimensional duality group if and only if $m \geqslant 2 n-1$. 
We conclude by discussing the case of robots on $K_{8}$. The corollaries above imply the following results:

\begin{tabular}{|c|l|}
\hline $\begin{array}{l}\text { Number of robots } \\
\text { (or strands) on } K_{8}\end{array}$ & $\begin{array}{l}\text { Dimension and connectivity } \\
\text { at infinity for } \overline{C_{m}\left(K_{8}\right)}\end{array}$ \\
\hline 1 or 7 & 1-dim'l and infinite \\
\hline 2 or 6 & 2-dim'l and one-ended \\
\hline 3 or 5 & $\begin{array}{l}\text { 3-dim'l and simply } \\
\text { connected at infinity }\end{array}$ \\
\hline 4 & $\begin{array}{l}\text { 4-dim'l but only simply } \\
\text { connected at infinity }\end{array}$ \\
\hline
\end{tabular}

\section{References}

[1] A. Abrams, Configuration spaces of colored graphs, Geom. Dedicata 92 (2002), no. 1, 185-194, Dedicated to John Stallings on the occasion of his 65 th birthday.

[2] A. Abrams and R. Ghrist, Finding topology in a factory: configuration spaces, Amer. Math. Monthly 109 (2002), no. 2, 140-150.

[3] C.A. Athanasiadis, Decompositions and connectivity of matching and chessboard complexes, Discrete Comput. Geom. 31 (2004), no. 3, 395-403.

[4] K. Barnett and M. Farber, Topology of configuration space of two particles on a graph. I, Algebr. Geom. Topol. 9 (2009), no. 1, 593-624.

[5] A. Björner, L. Lovász, S.T. Vrećica and R.T. Živaljević, Chessboard complexes and matching complexes, J. London Math. Soc. (2) 49 (1994), no. 1, 25-39.

[6] N. Brady, J. McCammond and J. Meier, Local-to-asymptotic topology for cocompact CAT(0) complexes, Topology Appl. 131 (2003), no. 2, 177-188.

[7] N. Brady and J. Meier, Connectivity at infinity for right angled Artin groups, Trans. Amer. Math. Soc. 353 (2001), no. 1, 117-132.

[8] D. Farley, Homology of tree braid groups, in Topological and asymptotic aspects of group theory, Contemp. Math. 394, 101-112, Amer. Math. Soc., Providence, RI, 2006.

[9] D. Farley and L. Sabalka, Discrete Morse theory and graph braid groups, Algebr. Geom. Topol. 5 (2005), 1075-1109.

[10] D. Farley and L. Sabalka, On the cohomology rings of tree braid groups, $J$. Pure Appl. Algebra 212 (2008), no. 1, 53-71.

[11] Ś.R. Gal, Euler characteristic of the configuration space of a complex, Colloq. Math. 89 (2001), no. 1, 61-67.

[12] R. Geoghegan, Topological methods in group theory, Grad. Texts in Math. 243, Springer-Verlag, New York, 2008.

[13] R. Ghrist, Configuration spaces and braid groups on graphs in robotics, in Knots, braids, and mapping class groups - papers dedicated to Joan S. Birman (New York, 1998), AMS/IP Stud. Adv. Math. 24, 29-40, Amer. Math. Soc., Providence, RI, 2001. 
[14] J. Jonsson, Five-torsion in the homology of the matching complex on 14 vertices, J. Algebraic Combin. 29 (2009), no. 1, 81-90.

[15] J. Jonsson, More torsion in the homology of the matching complex, Experiment. Math. 19 (2010), no. 3, 363-383.

[16] J. Jonsson, On the 3-torsion part of the homology of the chessboard complex, Ann. Comb. 14 (2010), no. 4, 487-505.

[17] V. Kurlin, Computing braid groups of graphs with applications to robot motion planning, Homology Homotopy Appl. 14 (2012), no. 1, 159-180.

[18] J. Shareshian and M.L. Wachs, Torsion in the matching complex and chessboard complex, Adv. Math. 212 (2007), no. 2, 525-570.

[19] M.L. Wachs, Topology of matching, chessboard, and general bounded degree graph complexes, Algebra Universalis 49 (2003), no. 4, 345-385. Dedicated to the memory of Gian-Carlo Rota.

[20] G.M. Ziegler, Shellability of chessboard complexes, Israel J. Math. 87 (1994), no. $1-3,97-110$.

John Meier meierj@lafayette.edu

Department of Mathematics, Lafayette College, Easton PA 18042, USA

Liang Zhang zhangl@lafayette.edu

Department of Mathematics, Lafayette College, Easton PA 18042, USA 